\title{
Exon organisation of the mouse gene encoding the Adrenoleukodystrophy related protein (ALDRP)
}

\author{
Cyril Broccardo ${ }^{1}$, Nathalie Troffer-Charlier ${ }^{2}$, Stephane Savary ${ }^{1}$, Jean Louis M andel ${ }^{2}$ and \\ Giovanna Chimini ${ }^{1}$ \\ ${ }^{1}$ Centre d'I Immunologie IN SE R M -CNRS de M arseille-L uminy, M arseille \\ IIG B M C Illkrich, France
}

\begin{abstract}
ALDR is one of the four genes encoding an ATP Binding Cassette (ABC) hemitransporter of the peroxisomal membrane so far identified in mammalian cells. The best known of these is X-ALD, whose dysfunction has been causally associated with X-linked adrenoleukodystrophy. ALDR and X-ALD protein product are closely related and we show here that this striking conservation is maintained at the genomic level. Although extending to a larger genomic region, the organisation of the mouse ALDR gene mirrors exactly that of $X$-ALD. This supports further the hypothesis that among the four known peroxisomal ABC hemi-transporters ALDRP is the most likely candidate as a modifier contributing to the phenotypic variability of X-linked adrenoleukodystrophy.
\end{abstract}

Keywords: adrenoleukodystrophy related gene; adrenoleukodystrophy; gene structure; ATP binding Cassette transporter

$X$-linked A drenoleukodystrophy is a severe demyelinating disorder associated with impaired catabolism of very long chain fatty-acids (VLCFA) ${ }^{1}$ and characterised by a high degree of phenotypic variability, even within a family. The molecular basis of the disease is the loss of function of a peroxisomal A BC (ATP Binding Cassette) hemi-transporter encoded by the ALD gene. ${ }^{2,3}$ The comparison with the inactivation of homologous genes in yeast suggests that the ALD protein (A LDP) may be involved in peroxisomal transport of activated VLCFA. ${ }^{4}$ A ttempts to generate a mouse

Correspondence: Giovanna Chimini, Centre d'Immunologie I N SE R M -CNR S de M arseille L uminy, Case 906, Parc Scientifique de Luminy, $13288 \mathrm{M}$ arseille Cedex 09, France. Tel: 0033 4 91269404; Fax: 00334 91269430; E-mail: chimini@ciml.univmrs.fr

R eceived 14 January 1998; revised 22 A pril 1998; accepted 19 M ay 1998 model for adrenoleukodystrophy by the knock out of the ALD gene were partially disappointing since no neurological abnormalities were detected. These mice express a biochemical phenotype somewhat milder than observed in human patients. ${ }^{5,6}$ This suggests that related hemi-transporters, potentially able to dimerise with ALDP in a functional complex, may play a modifier role in the final phenotypic outcome of the disease. In other biochemical pathways, heterodimerisation of hemi-transporters is known to have a key functional role. ${ }^{7,8}$ At least three other $A B C$ hemitransporters are located in the membrane of the peroxisome, but their combinatorial assembly in one or more functional transporters is yet unknown. ${ }^{9}$ They are encoded by the ALDR, PMP70 and PMP70-related genes. ${ }^{10-13}$ The A LDR protein (A LDRP) is the closest to ALDP with $66 \%$ amino acid identity, whereas 
PM P70 and PM P70R score at 35\% and 25\% identity with A LD P respectively. H ence, it seems reasonable to consider the ALDR gene a likely candidate as a modifier of the phenotype in mouse or in human patients carrying $A L D$ null mutations. The $A L D R$ gene ( $A L D L 1$ in the official GDB nomenclature) maps to human chromosome 12 and mouse chromosome $15 .^{14}$ In mouse, it is predominantly expressed in brain and adrenals ${ }^{10,15}$ in contrast to the rather ubiquitous expression of the ALD gene. We describe here the structural organisation of the $A L D R$ gene and extend the analysis of conservation between ALD and ALDR at the genomic level.

A mouse 129SV genomic library (Stratagene, La Jolla, CA , U SA, in lambda FIXII vector) was screened with the full length ALDR CDNA. Labelling of the probe by random-primed method, highly stringent hybridisation conditions and phage plate purification ${ }^{16}$ led to the isolation of eight individual phages ordered in two non-overlapping contigs. Phage overlaps were

A

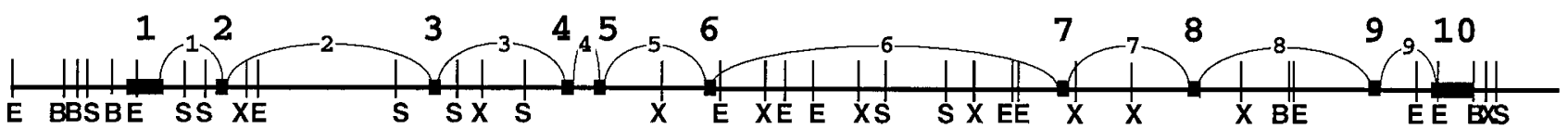
$1 \mathrm{~kb}$

B

ALDP M-PVLSTP--RPSRV-TTLKRTAVVLALTAYGVHKIYPLVRQCLT-PA-RGPQVPAGEPTQ-----EASGATATK--AGMNRVFLQRLLALLRLLFPRVL 87 ALDRP MIHMLNAAAYRVKWTRSGAAKRAACLVAAAYALKTLYPIIGKRLKOPGHRKAKAEAYSPAENREILHCTEIICKKPAPGLNAAFFKQLLELRKILFPKLV 100 TM1 TM2

ALDP CRETGLLALHSAALVSRTFLSVYVARLDGRLARCIVRKDPRAFSWQLIQWLLIALPATFINSAIRYLEGQLALSFRSRLVAHAYGLYFSQQTYYRVSNMD 187 ALDRP TTETGWLCLHSVALISRTFLSIYVAGLDGKIVKSIVEKKPRTFIIKLIKWLMIAIPATFVNSAIRYLECKLALAFRTRLVDHAYETYFANQTYYKVINMD 200 TM3 TM4

ALDP GRLRNPDQSLTEDVVAFAASVAHLYSNLTKPLLDVAVTSYTLLRAARSRGAGTAWPSAIAGLVVFLTANVLRAFSPKFGELVAEEARRKGELRYMHSRVV ALDRP GRLANPDQSLTEDIMMFSQSVAHLYSNLTKPILDVILTSYTLIRTATSRGASPIGPTLLAGLVVYATAKVLKACSPKFGSLVAEEAHRKGYLRYVHSRII

1 TM5 TM6 2.

ALDP ANSEEIAFYGGHEVELALLQHSYQDLASQINLILLERLWYVMLEQFLMKYVWSASGLLMVAVPIITATGYAESDSEAMKKAALEMKEEELVSERTEAFTI ALDRP ANVEEIAFYRGKKVEMKQLQKCYKALAYQMNLILSKRLWY IMIEQFLMKYVWSSCGLIMVAIPIITATGFADGDLEDGPK-------QAMVSDRTEAFTT 4.

ALDP ARNLLTAAADATERIMSSYKEVTELAGYTARVYEMFQVFEDVKHCRFKRTGDLEEAQAGPGVMVQSGVHVEGPLKIOGQVVDVEQGIICENIPIITPTGE

ALDRP ARNLLASGADAIERIMSSYKEITELAGYTARVYNMFWVFDEVKRGIYKRTVT-OEPENHSKRGGNLELPLSDTLAIKGTVIDVDHGIICENVPIITPAGE $A B C$

ALDP VVVASLNIRVEEGMHLLITGPNGCGKSSLFRILGGLWPTYSGVLYKPP PORMFYIPORPYMSVGSLRDOVIYPDSAEDMRRKGCSEOOLEATLGIVHLRH ALDRP VVASRLNFKVEEGMHLIITGPNGCGKSSLFRILSGLWPVYEGVLYKPPPQHMFYIPQRPYMSLGSLRDQVIYPDSADDMREKGYTDQDLERILHSVHLYH 7 8

ALDP ILQREGGWEAVCDWKDVLSGGEKQRIGMARMFYHRPKYALLDECTSAVSIDVEGKIFQAAKDAGIALLSITHRP SLWKYHTHLLQFDGEGGWKFEKLDSA ALDRP IVQREGGWDAVMDWKDVLSGGEKQRMGMARMFYHKPKYALLDECTSAVSIDVEGKIFQAAIGAGISLLSITHRPSLWKYHTHLLQFDGEGGWRFEQLDTA

Figure 1 a G enomic map of the $50 \mathrm{~kb}$ region encompassing the mouse A LDR gene. E coRI, Sacl, X bal and B amH I restriction sites are indicated. Exons and introns are numbered. b A lignment of murine ALD and ALDR protein showing the perfect conservation of splicing sites. The location of the transmembrane spanners (TM ) and the ATP B inding Cassette (A BC) are shown above the line. The last and first residues for each exon are underlined and hatched. Intron numbers are indicated 
identified by restriction mapping with four enzymes ( $\mathrm{E}$ coRI, X ba I, Sac I, BamHI) and hybridisation of phages with partial CDNA probes or specific oligonucleotides spaced along the coding sequence. Shotgun subcloning of the relevant phage fragments in pBSK $S$ vector (Stratagene, L a J olla, CA , U SA ) was carried out, allowing sequencing of the exon-introns boundaries in both directions (Pharmacia, B iotech, U ppsala, Sweden, T7 Polymerase sequencing kit on double-stranded templates). The size of the introns was estimated by PCR either on whole phages or their subclones and confirmed when possible on genomic DNA from 129SV mice. Oligonucleotides used as primers were derived from the cDNA sequence ( $17 \mathrm{bp}$ sequences starting from the following positions on the GB AC Z 48670: 918, 1220, 1331, 1510,1664, 1708, 1911, 2004, 2129 for the sense and 1314, 1423, 1596, 1640,1773, 1950, 2077, 2149, 2312 for the antisense primers) and chosen in order to allow the amplification reaction to be performed in standard conditions. The gap between the two sets of overlapping phages, estimated as smaller than $2 \mathrm{~kb}$ on the basis of genomic Southern analysis (not shown) with probes derived from the $3^{\prime}$ end of the CDNA could in fact be filled by PCR amplification on genomic D NA with a pair of primers targeted at the last two exons.

The resulting overall genomic map spans more than $50 \mathrm{~kb}$ (Figure 1a), and was confirmed by Southern blotting on genomic DNA (not shown). The ALDR gene is composed of 10 exons and 9 introns of highly

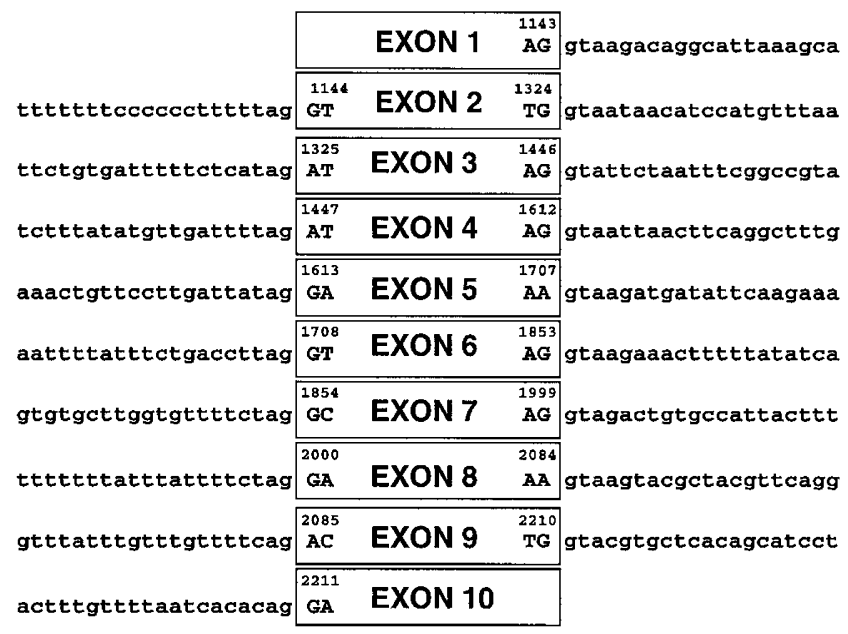

Figure 2 Exon-introns junctions for ALDR conform to consensus splice sites. Intron sequences flanking each exons are shown as well as the first and last two bases of the exons (in capitals). The position of each exon limit with respect to the cD NA sequence is indicated variable size. This structure mirrors exactly that of the human and mouse ALD genes ${ }^{17,18}$ with a perfect conservation of positions of splice sites (Figure $1 b$ ). The size of the coding sequence in exons $2-10$ is quite homogeneous, whilst the first exon encodes 313 amino acids ( $42 \%$ of the whole protein) and contains four out of the six predicted transmembrane segments (Figure $1 B$ ). The structure of the ALD and ALDR genes is very different from that of the PMP 70 gene, a more distant relative, with 23 exons $^{19}$ (G B AC \#X 83467 to \#X 83489). The mouse ALDR gene is larger than the mouse and human AL D genes (around $22 \mathrm{~kb}$ each); this is due to larger introns in particular the last four ${ }^{6-9}$ which span in ALDR more than $20 \mathrm{~kb}$ as compared with less than $4 \mathrm{~kb}$ in $A L D$. All the exon-intron junctions in the $A L D R$ gene conform to the known rules of splicing site (Figure 2 ).

The knowledge of the structure of the mouse A LDR gene will allow the engineering, by homologous recombination, of an A LDR knock-out mouse. This will be important since the recently constructed A LD knockout mice do not show neurologic abnormalities., ${ }^{5,6}$ The mouse $A L D$ and $A L D R$ genes show a very different pattern of expression, somewhat complementary in several organs. ${ }^{15} \mathrm{M}$ oreover, the mouse ALDR is much more highly expressed than its human counterpart, 20,10 in particular in adrenals and might thus play a more important role in VLCFA catabolism. A lthough the association of two homologous hemi-transporters into a functional complex has been assessed in mammals ${ }^{7}$ and in yeast, ${ }^{9}$ the facultative dimerisation with alternative partners has so far been reported only in the case of white, brown, and scarlet in D. melanogaster. ${ }^{8}$ The presence of several $A B C$ transporters on eukaryote peroxisomal membranes allows the hypothesis that tissue specific complexes, assembled from different subunits, exist. In this context, however, the role of $A L D R$ as a modifyer or facultative dimerisation partner remains still to be ascertained. These questions will hopefully be answered by comparing and crossing A LDR knock-out mice with ALD knock-out mice.

\section{Acknowledgements}

CB and NTC were supported respectively by an ARC and A FM grant. This work was supported by institutional grants from INSERM and CNRS, specific grants from LLNC and from EEC concerted action on peroxisomal leukodystrophy (J $L M)$. 


\section{References}

1 Aubourg P: Adrenoleukodystrophy and other peroxisomal diseases. Curr O pin G enet D ev 1994; 4: 407-411.

2 M osser J, D ouar A -M, Sarde CO et al: Putative X-linked adrenoleucodystrophy gene shares unexpected homology with A B C transporters. Nature 1993; 361: 726-730.

3 M osser J, L utz Y, Stoeckel ME et al: The gene responsible for adrenoleukodystrophy encodes a peroxisomal membrane protein. Hum M ol G enet 1994; 3: 265-271.

4 Hettema $E H$, van Roermund CWT, Distel $B$ et al: The $A B C$ transporter proteins Pat1 and Pat2 are required for import of long-chain fatty acids into peroxisomes of Saccharomyces cerevisiae. E M B O J 1996; 15: 3813-3822.

5 Kobayashi T, Shinnoh N, K ondo A, Y amada T: A drenoleukodystrophy protein deficient mice represent abnormality of very long fatty acid metabolism. B iochem Biophys Res Comm 1997; 232: 631-636.

$6 \mathrm{Lu} \mathrm{JF}$, Lawler A M, Watkins PA et al: A mouse model for $X$-linked adrenoleukodystrophy. Proc $N$ atl A cad Sci USA 1997; 94: 9366-9371.

7 K elly A, Powis SH, K err L -A et al: A ssembly and function of the two ABC transporter proteins encoded in the human major histocompatibility complex. Nature 1992; 355: 641-644.

8 E wart GD, Cannell D, Cox GB, Howells A : : M utational analysis of the traffic ATPase $(A B C)$ transporters involved in uptake of eye pigment precursors in D rosophila melanogaster. Implications for structure-function relationships. J B iol Chem 1994; 269: 10370-10377.

9 Shani N, Valle D: A Saccharomyces cerevisiae homolog of the human adrenoleukodystrophy transporter is a heterodimer of two half A TP-binding cassette transporters. P roc Natl A cad Sci USA 1996; 93: 11901-11906.

10 L ombard-Platet G, Savary S, Sarde CO, Mandel J L, Chimini G: A close relative of the adrenoleukodystrophy (ALD) gene codes for a peroxisomal protein with a specific expression pattern. Proc Natl A cad Sci USA 1996; 93: 1265-1269.
11 Kamijo K, Taketani S, Yokota S, O sumi T, H ashimoto T: The 70-kD a peroxisomal membrane protein is a member of the MDR (P-glycoprotein)-related ATP-binding protein superfamily. J Biol Chem 1993; 265: 4534-4540.

$12 \mathrm{H}$ olzinger $\mathrm{A}, \mathrm{K}$ ammerer $\mathrm{S}, \mathrm{R}$ oscher $\mathrm{A} \mathrm{A}$ : Primary structure of human PMP69, a putative peroxisomal ABCtransporter. Biochem Biophys Res Comm 1997; 237: 152-157.

13 Shani N, Jimenez-Sanchez G, Steel G, Dean M, Valle D: Identification of a fourth half A BC transporter in the human peroxisomal membrane. Hum M ol G enet 1997; 6: 1925-1931.

14 Savary S, Troffer-Charlier N, G yapay G, Mattei M G, Chimini G: Chromosomal localization of the adreno leukodystrophy related (A LDR) gene in man and mouse. E ur J H um G enet 1997; 5: 99-102.

15 Troffer-Charlier N, D oerflinger $N, K$ romer-M etzger $E$, Fouquet $F, M$ andel $J L$, A ubourg P: M irror expression of A drenoleukodystrophy and Adrenoleukodystrophyrelated genes in mouse tissues and human cell lines. E ur J Cell Biol 1998; 75: 254-264.

16 Sambrook J, Fritsch E F, M aniatis T: M olecular cloning: a laboratory manual 2nd edn. Cold Spring $\mathrm{H}$ arbor Laboratory Press: Cold Spring H arbor, NY, 1989.

17 Sarde CO, M osser J, K ioschis P et al: G enomic organization of the A drenoleukodystrophy gene. Genomics 1994; 22: 13-20.

18 K ennedy M A, R owland SA, M iller A L et al: Structure and location of the murine adrenoleukodystrophy gene. $\mathrm{G}$ enomics 1996; 32: 395-400.

19 G ärtner J, J imenez-Sanchez G, R oerig P, Valle D: G enomic organization of the 70-kDa peroxisomal membrane protein gene (PXMP1). G enomics 1998; 48: 203-208.

$20 \mathrm{H}$ olzinger $\mathrm{A}$, K ammerer $\mathrm{S}$, B erger J, R oscher $\mathrm{A} A$ : CD NA cloning and mR NA expression of the human A drenoleukodystrophy related protein (ALDRP), a peroxisomal A BC transporter. Biochem Biophys Res Comm 1997; 239: 261-264. 